\title{
Observation of magnetic reconnection and current sheets in the solar wind
}

\author{
Pablo R. Muñoz ${ }^{1}$, Abraham C.-L. Chian ${ }^{1,2}$, Rodrigo A. Miranda ${ }^{1,2}$, \\ and Michio Yamada ${ }^{2}$ \\ ${ }^{1}$ National Institute for Space Research (INPE), \\ São José dos Campos-SP 12227-010, Brazil \\ email: pmunoz@dge.inpe.br \\ ${ }^{2}$ Research Institute for Mathematical Sciences (RIMS), Kyoto University, \\ Kyoto 606-8502, Japan
}

\begin{abstract}
We apply single- and multi-spacecraft techniques to search for currents sheets in the solar wind during the ICME event of 21 January 2005, using the Cluster magnetic field data. Two large-scale currents sheets are detected at the leading boundary of the ICME ejecta using the single-spacecraft technique, which exhibit physical characteristics typical of magnetic reconnection exhausts in the solar wind.
\end{abstract}

Keywords. Sun: solar wind, coronal mass ejections (CMEs), magnetic fields

\section{Introduction}

Magnetic reconnection in a current sheet is a key dynamical process that converts magnetic energy into particle kinetic energy in space and astrophysical plasmas (Nishida, 2007; Priest, 2007). Retinò et al. (2007) developed a systematic technique to search for thin current sheets based on the simultaneous measurement of magnetic field by four Cluster spacecraft. Using this method in situ evidence of magnetic reconnection and associated energy release was found in the Earth's magnetosheath (Sundkvist et al., 2007). In addition, $\mathrm{Li}$ (2008) proposed a systematic procedure to look for the existence of current sheets in the solar wind based on two-point correlation measurement of magnetic field by a single spacecraft. This single-spacecraft technique succeeded to identify largescale current-sheet-like structures in the solar wind using one Cluster spacecraft (Li et al., 2008).

In this paper, we study current sheets and magnetic reconnection in the solar wind upstream of the Earth's bow shock during the Interplanetary Coronal Mass Ejection (ICME) event of 21 January 2005, based on Cluster data. Four regions of this ICME are identified by ACE and WIND (Foullon et al., 2007): (1) upstream region, (2) sheath region, (3) non-compressive density enhancement (NCDE) region, and (4) magnetic cloud. First, a systematic search of current sheets is performed in the first three regions detected by Cluster using both single- (Li, 2008) and multi-spacecraft (Retinò et al., 2007; Sundkvist et al., 2007) techniques. Our systematic search is capable of finding both large-scale and small-scale current sheets in all three regions of the ICME. Second, the technique proposed recently by Gosling et al. (2005) for observing magnetic reconnection exhaust in a current sheet in the solar wind was applied to two current sheets located at the leading boundary of the ICME ejecta. We analyze these two current sheets in detail and demonstrate their association with magnetic reconnection exhausts in the solar wind, with evidence of bifurcated current sheets and a jet of plasma flow linked to each current sheet. 


\section{Detection of current sheets}

A current sheet is a localized region of an electric current confined to a nearly twodimensional surface which is ubiquitous in a magnetized plasma. To confine the electric current into a surface, the magnetic field surrounding the current sheet must change its direction significantly across the sheet surface (i.e., with strong shear in the magnetic field vector B). Two methods of single-spacecraft (Li, 2008) and multi-spacecraft (Retinò et al., 2007; Sundkvist et al., 2007) are used to systematically search for current sheets in the ICME event of 21 January 2005, based on the Cluster magnetic field data. The interval studied includes the upstream region of the ICME shock, the ICME sheath region (marked by the vertical dashed lines) between the shock arrival (SA) and the leading boundary of the ejecta (SB), and a portion of the non-compressive density enhancement (NCDE) region when Cluster is in the upstream region of the Earth's bow shock (Foullon et al., 2007). In the single-spacecraft method of $\mathrm{Li}$ (2008), in order to determine the presence of current sheets in a given time series $\mathrm{B}(\mathrm{t})$ it is necessary to prove first that the integrated distribution function $\mathrm{F}(\theta, \mathrm{t})$ for the interval, representing the probability of finding the angle between $\mathbf{B}(t)$ and $\mathbf{B}(t+\tau)$ larger than $\theta$, scales linearly with the time separation $\tau$ when $\theta$ is larger than some critical angle $\theta_{0}$, i.e., $F(\theta, N \tau) \sim N F(\theta, \tau)$ where $N$ is an arbitrary integer greater than zero. The current sheets seen in the upperand middle-panels of Fig. 1 are detected by following the procedure of $\mathrm{Li}$ (2008), assuring that a linear behavior is clearly seen in all scales for different critical angles. Our results show that this single-spacecraft method is capable of detecting current sheets of any scale $\tau \gg \delta, \delta$ is the resolution of data. The top and middle panels show current sheets of largescale $(\tau \sim 120 \mathrm{sec})$ and small-scale $(\tau \sim 2 \mathrm{sec})$, respectively, using the Cluster-1 data. The upper-panel of Fig. 1 shows two large-scale current sheets, SB1 and SB2, associated with the leading boundary of the ejecta SB. For this event, the multi-spacecraft method was able to detect current sheets of small-scale $\tau \sim 1.1$ sec using four Cluster spacecraft

ICME Shock of 21 January 2005

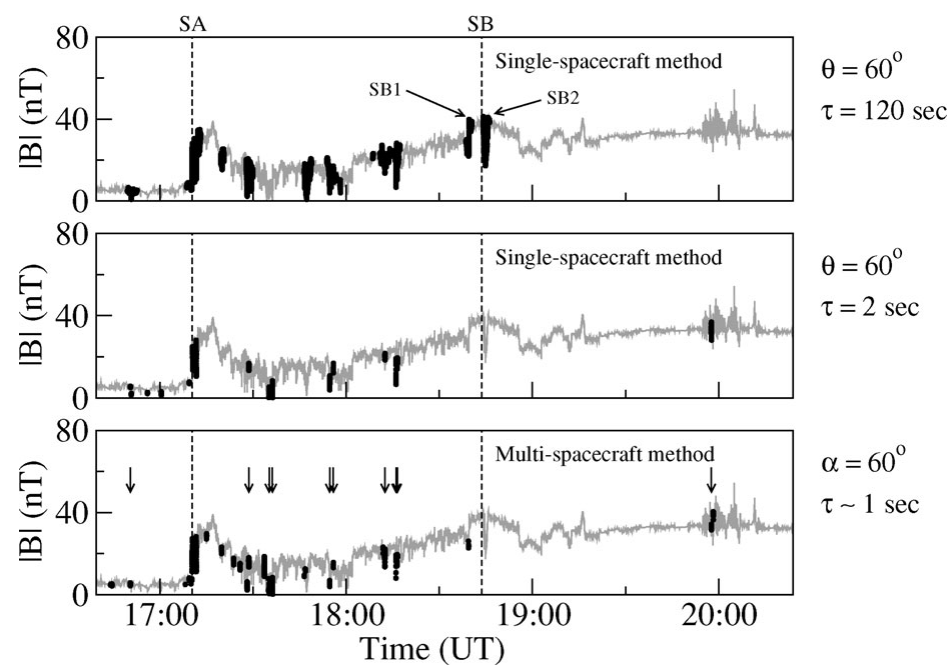

Figure 1. Detection of current sheets by single- and multi-spacecraft techniques using the magnetic field data of Cluster for the ICME shock of 21 January 2005. The gray line denotes the modulus of magnetic field $|B|$, and the black dots denote the current sheets detected. The arrows mark the current sheets detected by both single-spacecraft at $\tau=2 \mathrm{sec}$ and multi-spacecraft methods. SA denotes shock arrival, SB denotes the leading boundary of the ejecta where two large-scale current sheets SB1 and SB2 are found. 
(lower panel). The scale of the current sheets detectable by the multi-spacecraft method is given by $\tau=\Delta / V_{S W}$, where $\Delta$ is the minimum distance between $N$ spacecraft and $V_{S W}$ is the average speed of the solar wind with respect to the spacecraft. For this event, $\Delta \sim 933 \mathrm{~km}$ and $V_{S W} \sim 878 \mathrm{~km} / \mathrm{s}$. The arrows in the lower-panel of Fig. 1 indicate the currents sheets detected by both methods of single-spacecraft at $\tau=2$ sec (middle-panel) and multi-spacecraft (lower-panel).

\section{Observation of magnetic reconnection}

We discuss next the observation of magnetic reconnection exhausts in the ICME shock of 21 January 2005. Figure 2 displays the dynamics of magnetic reconnection processes associated with two current sheets, SB1 (left panels) and SB2 (right panels), located at the leading boundary of the ICME ejecta of Fig. 1. From top to bottom: the modulus of magnetic field $|B|(\mathrm{nT})$; three components of magnetic field $\left(B_{L}, B_{M}, B_{N}\right)$ in the current sheet (L, M, N) coordinates derived from the Minimum Variance Analysis (MVA) (Sonnerup and Cahill, 1967); the modulus of observed plasma velocity and the plasma velocity predicted by the reconnection theory of Sonnerup et al. (1981); three components of observed $\left(V_{L}, V_{M}, V_{N}\right)$ plasma velocity in LMN coordinates; the modulus of current density $|J|\left(\mathrm{nA} / \mathrm{m}^{2}\right)$ computed by the curlometer method based on multi-spacecraft Cluster data (Dunlop et al., 2002); three components of current density $\left(J_{L}, J_{M}, J_{N}\right)$. It is seen that $V_{L}$ is anti-correlated (correlated) with $B_{L}$ in the leading (trailing) edge of SB1 and $V_{L}$ is correlated (anti-correlated) with $B_{L}$ in the leading (trailing) edge of SB2. Such pairs of oppositely coupled changes in $\mathrm{V}$ and $\mathrm{B}$ are the signatures of magnetic

\section{Magnetic reconnection}
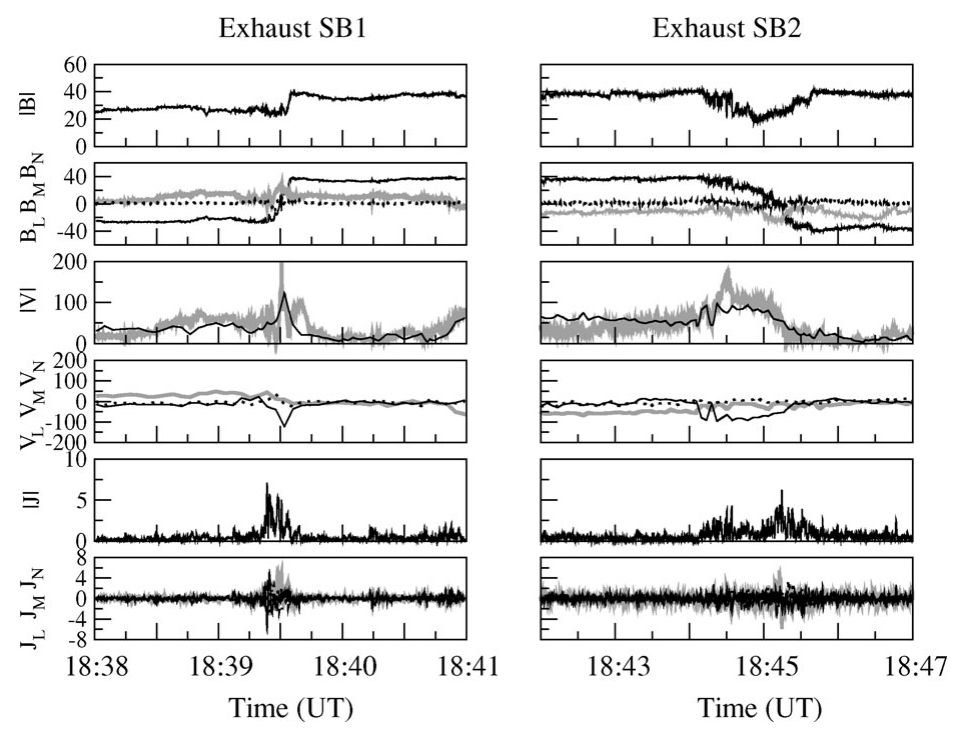

Figure 2. Observation of magnetic reconnection exhausts in two current sheets, SB1 and SB2, located at the leading boundary of the ICME ejecta of 21 January 2005, where $|B|(\mathrm{nT})$ is the modulus of magnetic field, $B_{L}$ (black), $B_{M}$ (gray) and $B_{N}$ (dotted) are the three components of the magnetic field in the $(L, M, N)$ coordinates, $V(\mathrm{~km} / \mathrm{s})$ is the modulus of observed plasma velocity (black) and the plasma velocity (gray) predicted by the reconnection theory of Sonnerup et al. (1981), $V_{L}$ (black), $V_{M}$ (gray) and $V_{N}$ (dotted) are the three components of plasma velocity, $|J|\left(\mathrm{nA} / \mathrm{m}^{2}\right)$ is the modulus of current density, and $J_{L}$ (black), $J_{M}$ (gray) and $J_{N}$ (dotted) are the three components of the current density. 
reconnection exhaust in bifurcated current sheets in the solar wind, as seen in the time series of $|J|$ (Gosling et al., 2005). Our three-dimensional analysis of magnetic field, current density and plasma velocity of two current sheets $S B 1$ and $S B 2$ are in agreement with the physical features of magnetic reconnection exhausts. The magnetic field vector $\mathbf{B}$ has a strong shear angle $\left(\theta_{S B 1} \sim 146^{\circ}\right.$ and $\left.\theta_{S B 2} \sim 150^{\circ}\right)$ between the leading and trailing edges of each current sheet. The current density vector $\mathbf{J}$ computed from the multispacecraft curlometer technique (Dunlop et al., 2002) shows a current sheet associated with the region of strong magnetic shear for both $S B 1$ and $S B 2$. In particular, each current sheet is associated with a strong jet of plasma flow resulting from a magnetic reconnection.

\section{Conclusion}

The solar wind provides a natural laboratory for observing coherent structures such as current sheets embedded in the interplanetary intermittent turbulence, upstream and downstream of an ICME. Phase synchronization due to nonlinear multiscale interactions is responsible for the formation of these coherent structures (Chian \& Miranda, 2009). Magnetic reconnection in current sheets plays an important role in energy dissipation in the solar wind turbulence. The methodology adopted in this paper to systematically detect current sheets and investigate the signatures of magnetic reconnection may deepen our understanding of the chaotic nature of the solar wind (Chian et al., 2006).

\section{Acknowledgement}

A. C.-L. Chian and R. A. Miranda wish to thank Professor Michio Yamada, Dr. Yoshitaka Saiki and RIMS at Kyoto University for their kind hospitality. This work is supported by CAPES and CNPq, Brazil.

\section{References}

Chian, A. C.-L., Kamide, Y., Rempel, E. L., \& Santana, W. M. 2006, J. Geophys. Res. 111, A07S03, doi:10.1029/2005JA011396.

Chian, A. C.-L. \& Miranda, R. A. 2009, Ann. Geophys. 27, 1789.

Dunlop, M. W., Balogh, A., Glassmeier, K. H., \& Robert, P. 2002, J. Geophys. Res-Space Phys. 107, A11, 1384.

Foullon, C., Owen, C. J., Dasso, S., et al.. 2007, Sol. Phys. 244, 139.

Gosling, J. T., Skoug, R. M., McComas, D. J., \& Smith, C. W. 2005, J. Geophys. Res.-Space Phys. 110, A01107.

Li, G. 2008, ApJ (Letters) 672, L65.

Li, G., Lee, E., \& Parks, G. 2008, Ann. Geophys. 26, 1889.

Nishida, A. 2007, in: Y. Kamide, Y. \& A. C.-L. Chian (eds.) Handbook of the Solar-Terrestrial Environment (Berlin, Springer), p. 280.

Priest, E. R. 2007, in: Y. Kamide \& A. C.-L. Chian (eds.) Handbook of the Solar-Terrestrial Environment (Berlin, Springer), p. 50.

Retinò, A., Sundkvist, D., \& Vaivads, A., et al. 2007, Nature Phys. 3, 236.

Sonnerup, B. U. O. \& Cahill, L. J., 1967, J. Geophys. Res. 72(1), 171.

Sonnerup, B. U. O. et al. 1981, J. Geophys. Res. 86,10049.

Sundkvist, D. Retinò, A., Vaivads, A., \& Bale, S. D. 2007, Phys. Rev Lett. 99, 025004. 